\title{
Individual Stress Vulnerability Is Predicted by Short-Term Memory and AMPA Receptor Subunit Ratio in the Hippocampus
}

\author{
Mathias V. Schmidt, ${ }^{1}$ Dietrich Trümbach, ${ }^{2}$ Peter Weber, ${ }^{1}$ Klaus Wagner, ${ }^{1}$ Sebastian H. Scharf, ${ }^{1}$ Claudia Liebl, ${ }^{1}$ \\ Nicole Datson, ${ }^{4}$ Christian Namendorf, ${ }^{1}$ Tamara Gerlach, ${ }^{1}$ Claudia Kühne, ${ }^{1}$ Manfred Uhr, ${ }^{1}$ Jan M. Deussing, ${ }^{1}$ \\ Wolfgang Wurst, ${ }^{1,2,3}$ Elisabeth B. Binder, ${ }^{1}$ Florian Holsboer, ${ }^{1}$ and Marianne B. Müller ${ }^{1}$ \\ ${ }^{1}$ Max Planck Institute of Psychiatry, 80804 Munich, Germany, ${ }^{2}$ Helmholtz Zentrum München/Technical University Weihenstephan, 85764 Neuherberg, \\ Germany, ${ }^{3}$ Deutsches Zentrum für Neurodegenerative Erkrankungen, 80336 München, Germany, and ${ }^{4}$ Leiden Amsterdam Center for Drug \\ Research/Leiden University Medical Center, 2300 RA Leiden, The Netherlands
}

Increased vulnerability to aversive experiences is one of the main risk factors for stress-related psychiatric disorders as major depression. However, the molecular bases of vulnerability, on the one hand, and stress resilience, on the other hand, are still not understood. Increasing clinical and preclinical evidence suggests a central involvement of the glutamatergic system in the pathogenesis of major depression. Using a mouse paradigm, modeling increased stress vulnerability and depression-like symptoms in a genetically diverse outbred strain, and we tested the hypothesis that differences in AMPA receptor function may be linked to individual variations in stress vulnerability. Vulnerable and resilient animals differed significantly in their dorsal hippocampal AMPA receptor expression and AMPA receptor binding. Treatment with an AMPA receptor potentiator during the stress exposure prevented the lasting effects of chronic social stress exposure on physiological, neuroendocrine, and behavioral parameters. In addition, spatial short-term memory, an AMPA receptor-dependent behavior, was found to be predictive of individual stress vulnerability and response to AMPA potentiator treatment. Finally, we provide evidence that genetic variations in the AMPA receptor subunit GluR1 are linked to the vulnerable phenotype. Therefore, we propose genetic variations in the AMPA receptor system to shape individual stress vulnerability. Those individual differences can be predicted by the assessment of shortterm memory, thereby opening up the possibility for a specific treatment by enhancing AMPA receptor function.

\section{Introduction}

Psychiatric disorders are a major health burden and affect millions of people worldwide (Kessler et al., 2003). Research on the biological underpinnings of affective disorders such as major depressive disorder (MDD) over the last decades has mainly focused on the monoamine hypothesis of depression (Charney, 1998), and currently available antidepressants are almost entirely supposed to increase monoamine signaling in the brain. However, a substantial proportion of patients does not respond adequately to pharmacological treatment (Huynh and McIntyre, 2008). In addition, the monoamine hypothesis has a number of limitations, including the inability to explain the lag phase of

Received Sept. 6, 2010; revised 0ct. 7, 2010; accepted 0ct. 14, 2010.

This work was supported by the Klinische Kooperationsgruppe of the Max Planck Institute of Psychiatry and Helmholtz Zentrum Muenchen, by the Federal Ministry of Education and Research in the framework of the National Genome Research Network [Grant 01 GS08151 (F.H., W.W.)], and by a grant from the Helmholtz Society (Helmholtz Alliance for Mental Health in an Ageing Society). We thank Daniela Harbich, Bianca Mayer, Carmen Sarnowski, and Pratik Talati for technical assistance and Jeffrey Witkin (Eli Lilly \& Co., Indianapolis, IN) for gratefully providing the AMPA receptor potentiator LY451646. Full microarray data are available at the GEO hybridization array data repository: http://www.ncbi.nlm.nih.gov/geo/query/acc.cgi?token=xjkthqikocyyirq\&acc=GSE11211.

The authors declare no competing financial interests.

Correspondence should be addressed to Dr. Mathias V. Schmidt, Max Planck Institute of Psychiatry, Research Group Neurobiology of Stress, Kraepelinstrasse 2-10, 80804 Munich, Germany. E-mail: mschmidt@mpipsykl.mpg.de.

DOI:10.1523/JNEUROSCI.4668-10.2010

Copyright $\odot 2010$ the authors $\quad 0270-6474 / 10 / 3016949-10 \$ 15.00 / 0$ therapeutic effects of current antidepressants, suggesting downstream adaptations as biologically relevant rather than the elevation of monoamine levels itself.

Research efforts on the biological foundations of MDD have therefore shifted toward the involvement of alternative neurotransmitter systems. There is increasing clinical and preclinical evidence for the involvement of the glutamatergic system in affective disorders (Sanacora et al., 2008; Hashimoto, 2009). Glutamate serum levels have been found to be increased in patients with MDD (Kim et al., 1982), and there is also a positive correlation between plasma glutamate levels and the severity of depressive symptoms (Mitani et al., 2006). There are two major types of ionotropic glutamate receptors: AMPA receptors and NMDA receptors (Rao and Finkbeiner, 2007). Strikingly, the noncompetitive NMDA receptor antagonist ketamine has been demonstrated to induce robust and rapid antidepressant effects in treatment-resistant depression (Zarate et al., 2006). Subsequently, a number of preclinical reports suggested that AMPA receptor activation is required for the antidepressant effects of ketamine (Maeng and Zarate, 2007; Maeng et al., 2008).

There are already a few indications that AMPA receptors may be critically involved in mood disorders (Alt et al., 2006). In animals, chronic treatment with the antidepressant imipramine increased hippocampal expression levels of the GluR1 subunit (Du et al., 2004), and the antidepressant tianeptine causes AMPA 
receptor phosphorylation, thereby possibly mediating its antidepressant-like actions (Svenningsson et al., 2007). In addition, acute treatment with the AMPA potentiator LY392098 induced antidepressant-like effects in the forced-swim test and also augmented the effects of several monoamine-based antidepressants (Li et al., 2003).

Although AMPA receptors have been described to be involved in mood disorders and antidepressant mechanisms of action (Bleakman et al., 2007), the intriguing question as to whether differences in AMPA receptor functioning might play a pivotal role in determining the individual vulnerability to stress and MDD has not yet been addressed. Chronic stress is one of the major risk factors for MDD (Wang, 2005), but it is still unclear why some individuals are highly vulnerable to stress exposure, while others show a remarkable level of resilience (Ozbay et al., 2008). Using a recently developed paradigm for individual stress vulnerability (Schmidt et al., 2009), here we tested the hypothesis of a causal involvement of AMPA receptors in stress vulnerability by combining evidence from genetic, pharmacological, and behavioral levels.

\section{Materials and Methods}

\section{Animal housing}

Male mice from the heterogeneous outbred mouse strain CD1 (Charles River Laboratories) were used for all experiments. Animals were 24-26 d old at the day of arrival and were kept on a $12 \mathrm{~h}$ light/dark cycle. Food and water werre provided ad libitum. The experiments were performed in accordance with European Communities Council Directive 86/609/EEC. All efforts were made to minimize animal suffering during the experiments. The protocols were approved by the committee for the Care and Use of Laboratory Animals of the Government of Upper Bavaria, Germany.

\section{Chronic stress paradigm}

The chronic social stress procedure was performed as described previously (Schmidt et al., 2007). In this paradigm, mice are exposed to a highly unstable social and hierarchical situation during the adolescence and young adult periods. Briefly, after a habituation period of $5 \mathrm{~d}$ after arrival, the group composition (four animals per cage) in each cage was changed twice per week for 7 weeks; each time, it was made sure that four mice from different cages were put together in a new, clean cage. The rotation schedule was organized to minimize the likelihood of a repeated encounter with the same mice throughout the experiment. Control mice remained with the same cage mates. All animals were single housed for 5 weeks after the 7 week stress procedure.

\section{Selection of stress-vulnerable and stress-resilient individuals}

Stress vulnerability or resilience was determined by assessing the basal morning corticosterone secretion 5 weeks after the end of the stress procedure, as described previously (Schmidt et al., 2009). In short, animals with the lowest $20 \%$ of corticosterone levels were regarded as stress resilient (fast recovering), whereas animals with the highest $20 \%$ of corticosterone levels were regarded as vulnerable individuals (slow recovering). For the initial analysis of AMPA receptor function, we only used animals identified as stress resilient or stress vulnerable under basal conditions 5 weeks after stress exposure. With stress vulnerability being a trait rather than a state, an additional group that never experienced chronic stress as "controls" is not always appropriate, as this group would contain both stress-vulnerable and stress-resilient individuals at unknown proportions. Therefore, in experiment 1 we only compared stress-vulnerable with stress-resilient individuals 5 weeks after stress exposure. All subsequent experiments, which do not rely on the selection of individual extremes in stress vulnerability, always contain an unstressed comparison group.

\section{Experimental design}

Experiment 1: stress vulnerability and AMPA receptor expression. We first investigated, whether animals identified as being either stress vulnerable or stress resilient differ in the expression and function of their AMPA receptor system. Therefore, 160 male $\mathrm{CD} 1$ mice were subjected to the chronic social stress procedure and subsequently recovered for 5 weeks. Based on corticosterone levels 5 weeks after stress exposure, 32 animals were identified as stress vulnerable (top $20 \%$ corticosterone), and 32 were identified as stress resilient (lowest $20 \%$ corticosterone). Of these, six animals per group were used to analyze the hippocampal expression levels of AMPA receptor subunits by in situ hybridization as well as for AMPA receptor binding analysis. Furthermore, six animals per group were used for a microarray expression profiling of the CA1 and the dentate gyrus regions of the hippocampus. The remaining animals were not used for analyses.

Experiment 2: modulation of stress vulnerability by enhancing AMPA receptor function. In this experiment, we subjected 128 animals to the chronic social stress procedure for 7 weeks, whereas another 80 animals were used as unstressed controls. During the last 4 weeks of the stress exposure, half of the animals received a daily subcutaneous injection of the AMPA potentiator LY451646 (Eli Lilly \& Co.), and the other half received vehicle injections. After a 4 week stress- and treatment-free period, all animals were subjected to the noveltyinduced suppression of feeding test. One week later, all animals were killed under basal conditions.

Experiment 3: prediction of individual stress vulnerability. Next, we investigated whether individual stress vulnerability might be a predictable trait. We therefore tested 140 male and 140 female CD1 mice at the age of $26 \mathrm{~d}$ in the Y-maze for short-term memory (STM) performance. To obtain groups of animals with a stable level of STM, we first selectively bred these CD1 mice for four generations, using STM as a selection criterion [low-STM (L-STM), $<35 \%$ spontaneous alternation; intermediate-STM (I-STM), 45-55\% spontaneous alternation; high-STM (H-STM), >70\% spontaneous alternation). The offspring of the fourth generation (F4) of these three lines were then subjected to the chronic social stress paradigm or treated as controls. During the last 4 weeks of the stress exposure, all animals received a daily subcutaneous injection of the AMPA potentiator LY451646 (Eli Lilly \& Co.) or vehicle. After a 4 week stress- and treatment-free period, all animals were subjected to the novelty-induced suppression of feeding test. One week later, all animals were killed under basal conditions. A separate cohort of animals of the L-STM and H-STM lines was used to determine hippocampal GluR1 and GluR2 expression at postnatal day 26 and at adulthood.

Experiment 4: genetic basis of individual stress vulnerability. We now assessed whether differences in individual stress vulnerability could be a genetic trait. From previous experiments using the same paradigm, we had DNA samples of 274 animals ( 74 controls, 200 stressed animals), of which we also measured corticosterone levels at the end of the stress procedure and after the 5 week recovery period. We then selected tagging single-nucleotide polymorphisms (SNPs) in the Grial gene encoding GluR1 and the Gria2 gene encoding GluR2 (see SNP selection and genotyping) from the Mouse Phenome Database SNP collection (http:// phenome.jax.org/SNP/) and tested their genotypes for association with the phenotypes of the mice.

\section{Sampling procedure}

Trunk or tail cut blood was collected individually in labeled $1.5 \mathrm{ml}$ EDTA-coated microcentrifuge tubes (Kabe Labortechnik). The time between the first disturbance of the animals and the sampling was, in all cases, $<1 \mathrm{~min}$. Tail blood was collected without anesthesia as described previously (Fluttert et al., 2000). For trunk blood collection, animals were anesthetized with isoflurane and decapitated. All blood samples were kept on ice and later centrifuged for $15 \mathrm{~min}$ at $6000 \mathrm{rpm}$ at $4^{\circ} \mathrm{C}$. Plasma was transferred to clean, labeled $1.5 \mathrm{ml}$ microcentrifuge tubes. All plasma samples were stored frozen at $-20^{\circ} \mathrm{C}$ until the determination of corticosterone by radioimmune assay (MP Biomedicals). To increase the assay sensitivity for basal samples with low-corticosterone content, samples were less diluted than described by the manufacturer, and the standard curve was extended by a low concentration standard. After decapitation, brains were removed, frozen in isopentane at $-40^{\circ} \mathrm{C}$, and stored at $-80^{\circ} \mathrm{C}$ for in situ hybridization. Adrenal glands were removed, dissected from fat, and weighed. 


\section{Gene expression analysis}

In situ hybridization was performed on cryosections as described previously (Schmidt et al., 2007). The antisense cRNA hybridization probes were between 400 and 500 base pairs long and complementary to the gene of interest. The following primers were used to generate cDNA hybridization probes: GluR1, CCGTTGACACATCCAATCAG (forward primer) and GGCTCCACTCTCCTTGAACTT (reverse primer), resulting in a hybridization probe of 404 base pairs; GluR2, CAGTTTCGCAGTCACCAATG (forward primer) and TCGCAGTCAAGGATTACACG (reverse primer), resulting in a hybridization probe of 429 base pairs. The slides were exposed to Kodak Biomax MR films (Eastman Kodak) and developed. Autoradiographs were digitized, and relative expression was determined by computer-assisted optical densitometry (Image 1.42; National Institutes of Health).

\section{AMPA binding}

Hippocampal sections were preincubated for $20 \mathrm{~min}$ in $50 \mathrm{~mm}$ Tris acetate buffer containing $100 \mathrm{~mm}$ potassium chloride and incubated for 30 min in the same buffer composition including $30 \mathrm{~nm}{ }^{3} \mathrm{H}$-AMPA (PerkinElmer). Nonspecific binding was assessed in the presence of $1 \mathrm{~mm}$ glutamate. Sections were washed in three changes of ice-cold buffer for a total of $60 \mathrm{~s}$ and rinsed in distilled water. Autoradiographic exposure time to Kodak Biomax MR films (Eastman Kodak) was $21 \mathrm{~d}$.

\section{Tissue dissection and expression profiling}

Tissue dissection was performed according to Datson et al. (2004) with some minor modifications. In short, brain slices of the hippocampal region were cut at 20 ' $6 \mathrm{dm}$ and thaw-mounted on membrane-coated slides (Carl Zeiss MicroImaging). Laser dissection of CA1 and dentate gyrus material was performed using a laser-capture microscope (Carl Zeiss MicroImaging). Extracted samples were immediately dissolved in 100 '6dl of TRIZOL (Invitrogen Life Technologies), and RNA was extracted according the manufacturer's instructions. RNA quality and quantity was checked by analyzing 1 ' $6 \mathrm{dl}$ of RNA on the 2100 Bioanalyzer using the RNA 6000 Pico LabChip kit (Agilent Technologies) according to the manufacturer's instructions. High-quality RNA samples were then amplified twice using the Amino Allyl MessageAmp RNA Amplification kit (Ambion).

Pooled amplified RNA samples were then hybridized on Illumina mouse BeadChips ( $n=6$ per group) and detected in the Illumina BeadArray Reader (Illumina). Gene expression was analyzed using the Illumina BeadStudio software (version 1.5.1.3). A differential score, which takes into account background noise and sample variability (Chudin et al., 2006), of \pm 50 and a fold regulation of \pm 1.5 were set as cutoff criteria. A differential score of 20 corresponds to a $p$ value of 0.01 . The data discussed in this publication have been deposited in the National Center for Biotechnology Information Gene Expression Omnibus (GEO; http:// www.ncbi.nlm.nih.gov/geo/) and are accessible through GEO Series accession number GSE11211.

\section{Bioinformatical analysis of gene expression data}

The expression profile of the complete cubic spline normalized raw data from the Illumina microarrays was represented by a heat map (see Fig. 1e). For the analysis of this heat map, we used the heatmap. 2 function with standard settings in the gplots package of $\mathrm{R}$ statistical software (http://www.r-project.org). Hierarchical clustering was applied to group experimental conditions (columns) as well as genes (rows) for the heat map. A dendrogram was shown only for the columns. Results were scaled by row and represented as $Z$ score.

To reveal the regulatory network of differentially expressed genes from the DNA microarray experiment, a list of significantly regulated genes in the CA1 region was mapped to the ResNet database of the Pathway Studio 5.0 software (Ariadne Genomics). The ResNet database stores biological relationships, especially interactions between gene products from all PubMed abstracts by using the Natural Language Processing Technology. To construct the network, we searched for direct interactions among the regulated genes restricting to the relationships geneexpression and promoter-binding. Within the network, gene products were represented as nodes, and the biological relationship between nodes was represented as an edge. Nodes are displayed using different shapes that represent the functional class of the gene product.

Regulated genes were imported into the BiblioSphere software (version 7.13; Genomatix) and mapped to Gene Ontology (GO) trees to identify their biological function. For identification of overrepresented GO terms, the BiblioSphere software calculates a $Z$ score for each term. The $Z$ score represents the difference between observed and expected annotations and is normalized to the SD of a hypergeometric distribution. GO terms with a $Z$ score $>1.96$ (which corresponds to a $p$ value of $0.05)$ are statistically significant.

\section{AMPA potentiator treatment}

The AMPA potentiator LY451646 (Eli Lilly \& Co.) was dissolved in saline containing $0.0025 \%$ Methocel (Georg Breuer). Mice were subcutaneously injected daily with LY451646 $(0.125 \mathrm{mg} / \mathrm{kg}$ body weight $)$ or saline during the last 4 weeks of stress exposure. During the recovery period of 5 weeks after the stress exposure, the animals were not treated.

To verify drug availability in the brain, LY451646 concentrations in plasma and the brain were measured using liquid chromatography-mass spectrometry/mass spectrometry (LC-MS/MS) $1 \mathrm{~h}$ after a subcutaneous injection in a separate pilot study. Brains were weighed and homogenized in a fivefold volume of ice-cold PBS containing EDTA and Trasylol using a Dispomix Drive (Biolabproducts). Before the chromatographic analysis, $100 \mu \mathrm{l}$ of plasma samples diluted with $100 \mu \mathrm{l}$ of $0.9 \% \mathrm{NaCl}$ or $200 \mu \mathrm{l}$ of brain homogenate samples were deproteinized by addition of 500 $\mu \mathrm{l}$ of acetonitrile [containing $100 \mathrm{ng} / \mathrm{ml}$ internal standard (IS)], vortexed for $120 \mathrm{~s}$, and centrifuged at $14,000 \times g$ at $0^{\circ} \mathrm{C}$ for $15 \mathrm{~min}$. Twenty microliters of the supernatant were mixed with $180 \mu \mathrm{l}$ of water by vortex, and $10 \mu \mathrm{l}$ aliquots of the resultant mixture were injected onto the LC-MS/MS system.

Samples for the calibration curves were prepared by adding different amounts of LY451646 into blank brain homogenate or blank plasma. All samples, including calibration curve, were processed in the same manner.

LC-MS/MS consisted of an 1100 HPLC system (Agilent Technologies) and a 4000 triple quadrupole mass spectrometer (Applied Biosystems) equipped with a Turbo Ion Spray ionization interface in positive multiple reaction monitoring mode. Chromatographic separation was performed on a Gemini C18 $(50 \times 2.0 \mathrm{~mm}$; Phenomenex $)$ with a particle size of 5 $\mu \mathrm{m}$. The mobile phase was composed of methanol-water $(70: 30, \mathrm{v} / \mathrm{v})$ containing $0.1 \%$ formic acid and $10 \mathrm{~mm}$ ammonium formate. Isocratic separation was performed with a flow rate of $0.2 \mathrm{ml} / \mathrm{min}$ and a run time of $5 \mathrm{~min}$. Column oven temperature was adjusted to $40^{\circ} \mathrm{C}$, and the injection volume was $10 \mu \mathrm{l}$. Deuterium-labeled cortisol (cortisol-9, -11, -12, $-12-d_{4}$ ) was selected as the IS. Ion detection was based on monitoring [M $+\mathrm{H}]^{+}$ions in the analyte and IS in the first quadruple and their corresponding product ions in the third quadruple with a dwell time of 1000 and $100 \mathrm{~ms}$, respectively. The column effluent was monitored at the following transitions: LY451646 $\mathrm{m} / z 343.2 \rightarrow 220.2$ and the internal standard cortisol- $d_{4} 367.2 \rightarrow 121.1$. General adjustment of the instrument was as follows: turbo spray temperature was set to $500^{\circ} \mathrm{C}$, ionization voltage was $5000 \mathrm{~V}$, and nitrogen gas adjustment was set to 70 and $40 \mathrm{U}$ for gases 1 and 2. The curtain gas was set to 15 , and the collisionally activated dissociation gas was set to $6 \mathrm{U}$. Entrance potential was $10 \mathrm{~V}$. The declustering potential, collision energy, and collision cell exit potential were set at 36, 13, and $12 \mathrm{~V}$ for LY451646 and at 71, 35, and $10 \mathrm{~V}$ for IS, respectively.

Data acquisition was performed with Analyst 1.4.2 software (MDSSCIEX), and peak area ratios of LY451646 to IS were plotted versus nominal concentration to generate a calibration curve for quantification.

\section{Behavioral analysis}

All behavioral tests were scored using a video-tracking system (Anymaze 4.20; Stoelting). Short-term spatial memory was tested recording spontaneous alteration behavior in the Y-maze as described previously (Simoes et al., 2007). The Y-maze was made of gray polyvinyl chloride and consisted of three arms with an angle of $120^{\circ}$ between each of the two arms differentially marked by tape symbols (triangles, bars, and plus signs, respectively). The arms were $30 \mathrm{~cm}$ (length) $\times 10 \mathrm{~cm}$ (width) $\times 15$ $\mathrm{cm}$ (height) each and evenly illuminated during testing (40 lux). Each 
mouse was placed in the center of the maze at the age of $26 \mathrm{~d}$ before the start of the stress paradigm and was allowed to explore all three arms freely for a period of $5 \mathrm{~min}$. Three consecutive choices of all three arms were counted as an alteration. Thus, the percentage of alternation was determined by dividing the total number of alterations by the total number of choices minus 2. Higher percentages of correct alterations are interpreted as better STM.

Anxiety-related behavior was assessed using the novelty-induced suppression of feeding test based on the work of Merali et al. (2003). Animals received a piece of almond in their home cage for 2 consecutive days $3 \mathrm{~d}$ before testing. The consumption time of the almond in the home cage environment was $<30 \mathrm{~s}$ for all mice on the second day, with no differences between the groups. Testing was then performed in an open-field arena as described below (light intensity, 50 lux), with a small piece of almond put in the center. The animals were familiar with the almond but were unfamiliar with the open-field arena. The latency until the initiation of food intake was recorded. The total test time was $30 \mathrm{~min}$. Shorter consumption times are interpreted as lower anxiety levels. In addition, we analyzed the locomotor activity of the animals in the open-field arena. The adaptation of locomotor activity over time, measured by distance traveled in the first minute minus distance traveled in the sixth minute, was used as an additional measure of anxiety (high adaptation equals low anxiety).

\section{SNP selection and genotyping}

SNP genotypes of 18 different strains [129S1/SvImJ, A/J, AKR/J, BALB/ cByJ, BTBR T+tf/J, C3H/HeJ, C57BL/6J, CAST/EiJ, DBA/2J, FVB/NJ, KK/HlJ, MOLF/EiJ, NOD/ShiLtJ, NZW/LacJ, PWD/PhJ, WSB/EiJ, 129X1/SvJ, CZECHII/EiJ including 16 from phase 1 resequencing by Perlegen (http://mouse.perlegen.com/mouse/index.html)] were downloaded from the SNP site of the Mouse Phenome Database (http://phenome. jax.org/SNP/). All known SNPs within $5 \mathrm{~kb} 5^{\prime}$ and $3^{\prime}$ of the loci encoding GluR1 (Gria1; NM_008165) on chromosome 11 and GluR1 (Gria2; NM_013540) on chromosome 3 were downloaded (supplemental Fig. $4 a, b$, available at www.jneurosci.org as supplemental material). Linkage disequilibrium structure, haplotypes, and tagging SNPs were determined using Haploview software version 3.32. Selection of tagging SNPs was performed using the Tagger algorithm implicated in this software with pairwise tagging and a required $R^{2}=1.0$. For GluR1, 1780 SNPs were selected, and 800 passed the selection threshold for $\geq 75 \%$ genotyped strains and Hardy-Weinberg equilibrium test $p$ value $\leq 0.001$. These were captured by 24 tagging SNPs at $R^{2}=1.0$. For GluR2, 662 SNP were selected, of which 392 passed filtering as described above, and these were tagged by 10 SNPs at $R^{2}=1.0$.

These 34 SNPs were genotyped in 274 CD1 animals on a MALDI-TOF (matrix-assisted laser desorption/ionization time-of-flight) mass spectrometer using Sequenom iPLEX technology (MassARRAY system; Sequenom). We used the PreXTEND-SNP Validation/Amplicon Design, the multiplexed hME Assay Design, and the PleXTEND-Multiplexed Assay Validation software modules. Genotype calls were made using the ArrayTyper 3.4 software.

\section{Statistical analysis}

The commercially available program SPSS 17 was used for statistical analysis. For multifactorial data sets, two- or three-way ANOVA was performed, followed by Bonferroni post hoc analysis in the case of three groups or two-tailed unpaired $t$ tests in the case of two groups. Simple group comparisons were performed using the two-tailed unpaired $t$ test. Correlations were analyzed using the Pearson correlation coefficient. The level of statistical significance was set at $p<0.05$. For SNP associations and interactions, additive genetic models were tested, with the level of statistical significance set at $p<0.05$ for main effects and $p<0.1$ for interactions. Four tagging SNPs were tested so that after Bonferroni correction, $p$ values of 0.0125 for main effects and 0.025 for interaction term were considered significant. Data are presented as mean \pm SEM.

\section{Results}

\section{Stress vulnerability correlates with a different AMPA receptor expression}

We have recently demonstrated that animals, which fail to recover after exposure to chronic social stress during their adolescence and early adulthood, can be used as a model of stress vulnerability (Schmidt et al., 2009). Utilizing this innovative approach, we first investigated whether stress-vulnerable and -resilient animals differ in their expression of AMPA receptor subunits. In situ hybridization revealed a significantly lower mRNA expression of the AMPA receptor subunit GluR1 in the CA1 and dentate gyrus of the hippocampus of stressvulnerable animals compared with stress-resilient individuals (Fig. 1a). In contrast, mRNA expression of the AMPA receptor subunit GluR2 was higher in the CA1 and dentate gyrus of stress-vulnerable compared with stress-resilient animals (Fig. $1 b)$. In addition, overall AMPA receptor binding measured with radioligand autoradiography revealed a significantly higher hippocampal binding in vulnerable compared with resilient animals (Fig. 1c).

In addition to these hypothesis-driven approaches, we also analyzed the hippocampal gene expression pattern between vulnerable and resilient animals. To investigate the mechanisms underlying the observed differences in individual stress vulnerability, we laser dissected the CA1 and dentate gyrus regions of the hippocampus of six vulnerable and six resilient mice 5 weeks after stress and performed a gene expression profiling analysis (GEO accession number GSE11211). An unsupervised hierarchical cluster analysis of all genes presented on the microarray revealed a clear distinction between the brain regions and also the experimental groups (Fig. 1d), supporting our previous data that animals vulnerable or resistant to chronic stress form distinct biological subgroups that can be identified by a specific gene expression profile (Schmidt et al., 2009). Moreover, gene ontology analysis of the regulated genes showed several biological functions that are statistically overrepresented in at least one of the two analyzed brain regions. One of these functional gene ontology classes, synaptic transmission, also included the AMPA receptor subunits GluR1 and GluR2 (Fig. 1e), confirming our in situ hybridization results. These results were reflected in the pathway analysis of the CA1 region, where the AMPA receptor subunits and interacting proteins came up in the main pathway cluster of regulated genes (supplemental Fig. 1, available at www.jneurosci.org as supplemental material).

\section{Individual stress vulnerability can be modulated by pharmacological AMPA receptor potentiation}

We now proceeded to test the hypothesis that differences in hippocampal AMPA receptor expression may contribute to the stress vulnerability or resilience of the animals. The higher GluR2-to-GluR1 subunit ratio in stress-vulnerable individuals suggested an increased availability of GluR2-containing AMPA receptors in the cell membrane of these animals. It has been reported extensively that the GluR2 subunit is a rate-limiting factor for the calcium influx after activation of these receptors, resulting in a desensitization of GluR2-containing AMPA receptors (for review, see Isaac et al., 2007). To pharmacologically counteract this effect in vulnerable individuals, we chose a treatment with the novel AMPA receptor potentiator LY451646 (Eli Lilly \& Co.), which has been shown to increase BDNF expression and neurogenesis in the hippocampus after acute and chronic treatment (Mackowiak et al., 2002; Bai et al., 2003), comparable to most antidepressants. To test the pharmacokinetic properties of this 

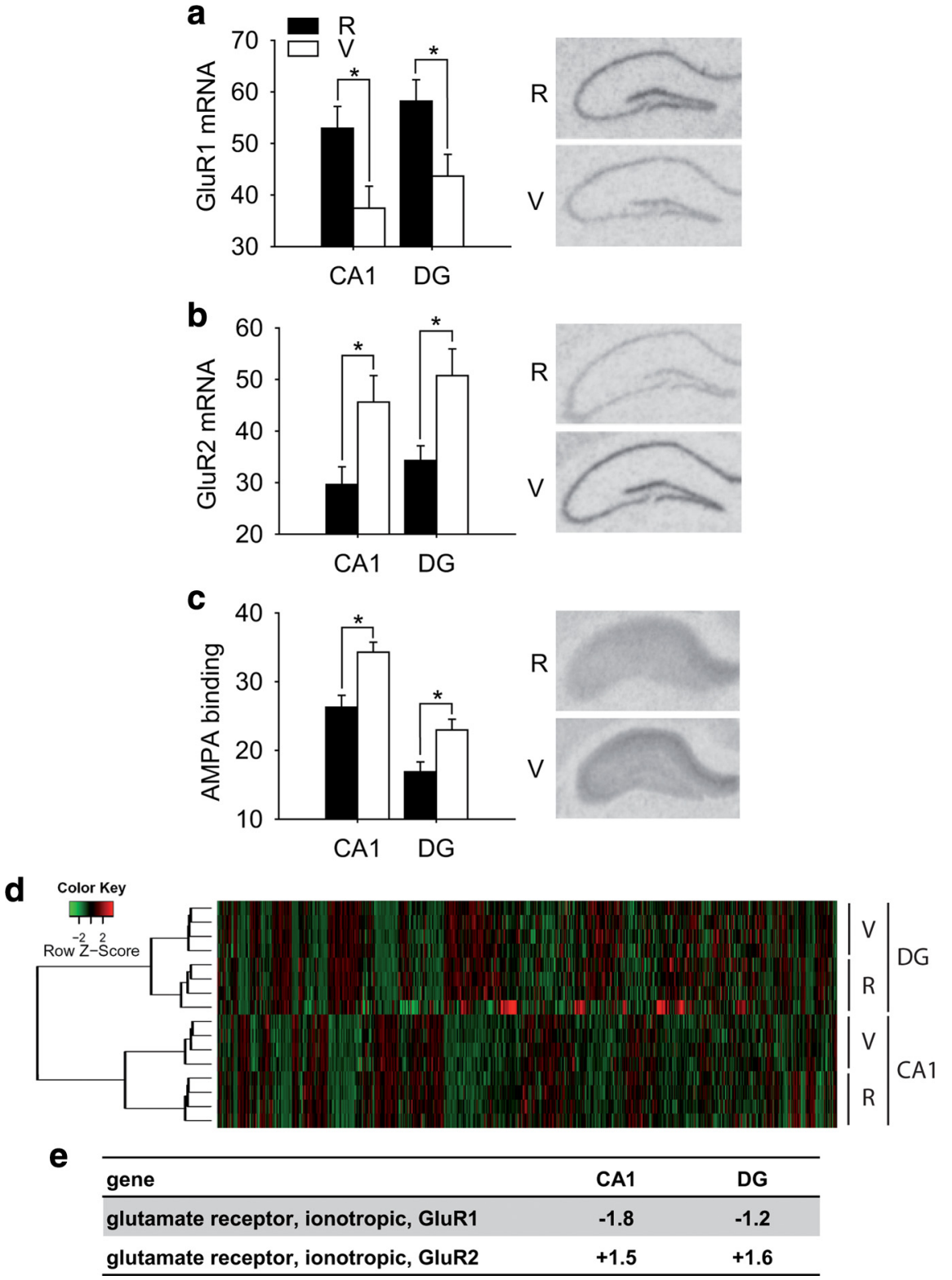

Figure 1. Comparison of AMPA receptor expression and function in vulnerable (V) and resilient (R) animals. $\boldsymbol{a}$, A significantly lower mRNA expression of the AMPA receptor subunit GluR1 was observed in vulnerable compared with resilient animals (CA1, $t_{(10)}=2.588, p<0.05$; dentate gyrus, $\left.t_{(10)}=2.456, p<0.05\right)$. Right, Autoradiographic sample pictures of GluR1 mRNA $(n=6)$. $\boldsymbol{b}$, In contrast, $m R N A$ expression of the AMPA subunit GluR2 was significantly increased in animals with a high stress vulnerability $\left(C A 1, t_{(10)}=2.596, p<0.05 ;\right.$ dentate gyrus, $\left.t_{(10)}=2.788, p<0.05 ; n=6\right)$. $C$, Animals with the vulnerable phenotype display an enhanced AMPA receptor binding compared with the stress-resilient animals $\left(C A 1, t_{(10)}=3.468, p<0.01\right.$; dentate gyrus, $\left.t_{(10)}=2.83, p<0.05 ; n=6\right)$. $\boldsymbol{d}$, Gene expression microarrays were performed using tissue of the CA1 and the dentate gyrus region of vulnerable and resilient animals 5 weeks after chronic stress exposure. An unsupervised hierarchical cluster analysis of all genes present on the array clearly distinguished the hippocampal subregions and also gene expression profiles of animals vulnerable and resilient to chronic stress. The heat map next to the dendrogram shows the expression profile of the genes on the microarrays. The green (lower expression) to red (higher expression) color scale indicates the normalized expression values of the probes (see color key). $\boldsymbol{e}$, Fold regulations of vulnerable versus resilient animals based on the microarray results for the GluR1 and Glur2 gene in the two different hippocampal subregions. Differential scores are as follows: GluR1 CA1, -204; GluR1 DG, -24; GluR2 CA1, 371; GluR2 DG, 206. R, Resilient; V, vulnerable; DG, Dentate gyrus. *Significant difference.

novel drug, we first showed that LY451646 crosses the bloodbrain barrier after a single subcutaneous injection (supplemental Fig. $2 a$, available at www.jneurosci.org as supplemental material). In the actual experiment, we treated animals with the AMPA receptor potentiator LY451646 or vehicle during the last 4 weeks of chronic stress exposure. At the end of stress exposure, animals from the chronic stress group showed significantly higher corti- costerone levels compared with controls; no significant differences were observed between AMPA potentiator- and vehicletreated animals, indicating that both groups were equally stressed during stress exposure (supplemental Fig. $2 b$, available at www.jneurosci.org as supplemental material). When examined 5 weeks after the termination of the stress procedure, vehicle-treated animals still had significantly elevated basal plasma concentrations of corticosterone compared with controls. However, corticosterone levels in AMPA potentiator-treated stress animals were significantly lower compared with vehicle-treated stress animals (Fig. $2 a)$, indicating that AMPA receptor potentiator treatment during stress promoted the recovery from the stress exposure and decreased stress vulnerability. Attenuation of hypothalamic-pituitaryadrenal (HPA) system hyperactivity after treatment with LY451646 was also reflected in the adrenal weight, indicating a lasting effect on corticosterone secretion (Fig. 2b). The observed differences between AMPA receptor potentiator- and vehicle-treated animals are mainly caused by the changes in the upper $20 \%$ of corticosterone secretors, the stress-vulnerable subpopulation of animals. In contrast, little or no effect of treatment was observed in the lower $20 \%$ stress-resilient group (supplemental Fig. $2 c$, available at www. jneurosci.org as supplemental material).

To further characterize the effect of AMPA receptor potentiation, we also tested the animals with respect to anxietyrelated behavior, one of the core symptoms of depression-like behavior in rodents. We have previously shown that exposure to chronic social stress during adolescence persistently increases anxietylike behavior in the novelty-induced suppression of feeding paradigm (Schmidt et al., 2007), an effect that is predominantly attributable to the increased anxiety in stress-vulnerable animals (Schmidt et al., 2009). Here we demonstrate that this effect could be completely prevented by treatment with the AMPA receptor potentiator LY451646 during the last 4 weeks of stress exposure (Fig. 2c). In addition, the adaptation of locomotor activity in a new environment, which is also a clear indicator of general anxiety, was significantly decreased in vehicle-treated stress animals but not in AMPA receptor potentiator-treated animals (Fig. 2d).

\section{Individual STM predicts stress vulnerability}

We now hypothesized that if alterations in AMPA receptor function are a heritable trait, they should be detectable as a behavioral or cognitive phenotype before the stress exposure. As AMPA re- 
ceptors are crucially involved in STM function (Izquierdo et al., 2002; Schmitt et al., 2005), we tested the hypothesis that natural variation in STM correlates with individual stress vulnerability. We therefore screened 140 male and 140 female CD1 mice at the age of 26-28 $\mathrm{d}$ for their spatial STM function using spontaneous alternation behavior in the Y-maze. The behavioral distribution showed a normal Gaussian distribution, with the majority of animals displaying an intermediate STM (Fig. 3a). We then performed a selective breeding approach starting from this CD1 founder population, where we always selected animals from the lowest, intermediate, or highest performance in STM as founders for the following generation. After four generations, the phenotype of the offspring of the three different breeding lines showed a distinct and linespecific phenotype (Fig. 3b). To assess whether differences in STM correlate with AMPA receptor expression and may therefore be predictive of stress vulnerability, we tested L-STM and H-STM animals at the age of 4 weeks and 3 months for hippocampal expression of GluR1 and GluR2. Whereas differences in STM did not correlate with GluR1 expression (data not shown), L-STM animals had a significantly higher GluR2 expression in the CA1 and dentate gyrus of the hippocampus at both examined ages (Fig. $3 c, d$ ).

The F4 offspring of the three breeding lines was then subjected to the chronic social stress procedure or kept undisturbed as controls. In addition, half of the animals were again treated during the last 4 weeks of stress exposure with the AMPA receptor potentiator LY451646, whereas the other animals received vehicle injections. At the end of the stress procedure, morning corticosterone levels were significantly increased in stress animals, with no effect of either STM line or treatment (Fig. 3e). Thus, chronic stress enhanced basal HPA axis activity regardless of genetic risk or pharmacological treatment. In contrast, after 5 weeks of recovery under stress- and drug-free conditions, only animals from the L-STM group still showed significantly increased basal corticosterone levels, whereas animals from the H-STM group had fully recovered (Fig. $3 f$ ). In addition, although AMPA receptor potentiator treatment significantly reduced corticosterone levels in formerly stressed L-STM animals, no effect of treatment was observed in I-STM or H-STM animals. Strikingly, whereas corticosterone levels in vehicle-treated animals directly after stress did not correlate with STM before stress exposure (supplemental Fig. 3, available at www.jneurosci.org as supplemental material), there was a significant negative correlation between STM performance at postnatal day 26 and basal corticosterone levels after 5 weeks of recovery from the chronic stress procedure (Fig. $3 \mathrm{~g}$ ). Thus, individuals with a poor short term memory at postnatal day 26 displayed higher corticosterone levels following recovery from chronic social stress than animals with a good STM.

We also observed a pronounced line difference with regard to anxiety-related behavior. Chronic stress exposure resulted in a b
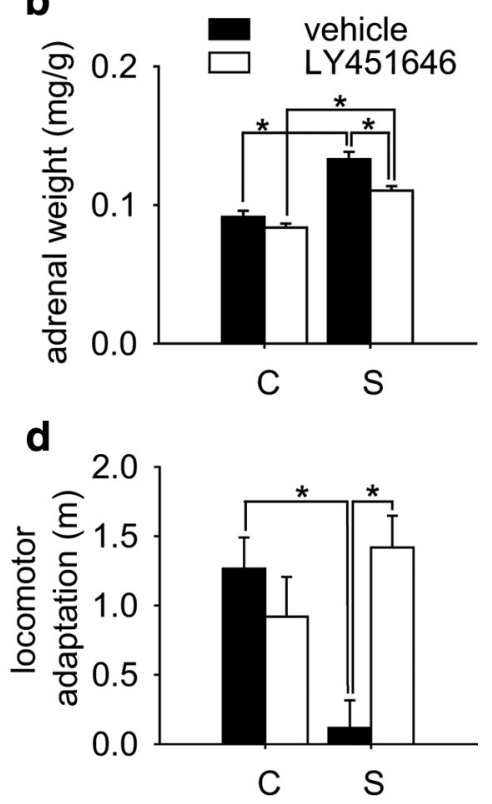

Figure 2. AMPA receptor potentiation during the last 4 weeks of stress exposure affects recovery from chronic social stress. $\boldsymbol{a}$, onen-field environment compared with controls. The increase in anxiety-related behavior was prevented by AMPA prevented by LY451646 pretreatment (treatment: $F_{(1,99)}=4.008, p<0.05$; condition $\times$ treatment interaction: $F_{(1,99)}=11.921$, $p<0.001)$. C, Control; S, stress. *Significant difference.

significant increase in anxiety-related behavior as measured with the novelty-induced suppression of feeding paradigm only in L-STM and I-STM animals, whereas there was no effect of the stress exposure in H-STM individuals (Fig. 3h). Furthermore, treatment with LY451646 significantly reduced anxiety-related behavior in the L-STM and I-STM stress groups but had no additional effect in the H-STM animals.

\section{A genetic polymorphism in the GluR1 gene correlates with individual stress vulnerability}

Finally, we tested whether genetic polymorphisms in the GluR1 or GluR2 gene are associated with individual stress vulnerability. Of 24 selected SNPs in the GluR1 gene, 10 turned out to be polymorphic in this sample, and 1 failed genotyping. For the GluR2 gene, 3 of the 10 selected SNPs were polymorphic, and 1 failed genotyping. Of these 13 polymorphisms, only 10 had a minor allele frequency of $\geq 5 \%$ and were thus included in the analysis (supplemental Table 1, available at www.jneurosci.org as supplemental material). We then analyzed the linkage disequilibrium structure of the common polymorphic SNPs in this sample using haploview. For GluR1, the seven SNPs give rise to three haplotypes and cluster in two bins, tagged by rs26962886 and rs26978382 (Fig. 4a,b). For GluR2, the three polymorphic SNPs are clustered in two bins tagged by rs30163792 and rs32567824. We then analyzed the interaction of these four SNPs and chronic stress exposure on corticosterone levels at the end of the stress 
a

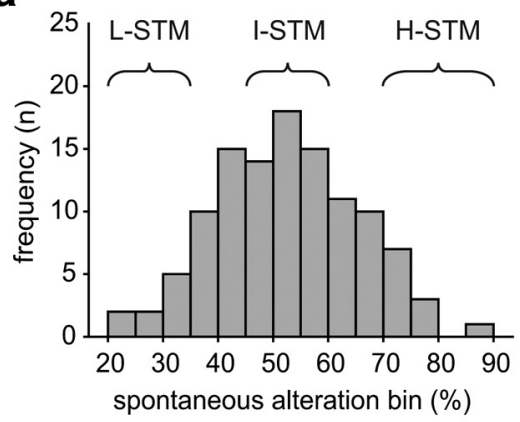

C
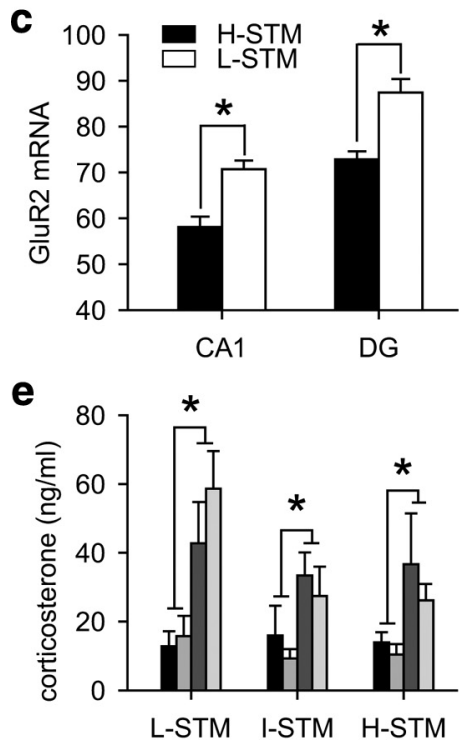

g

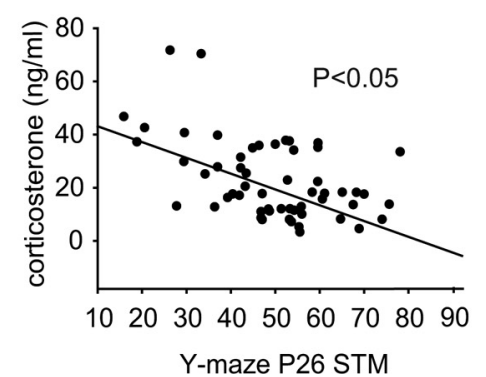

b

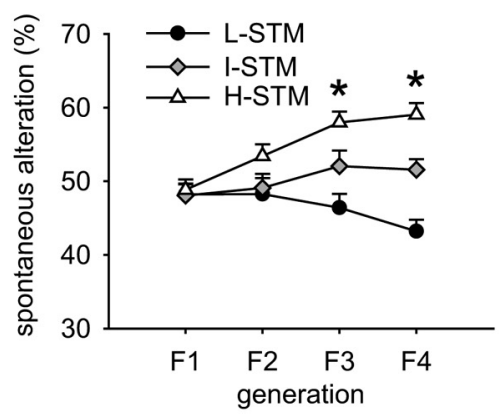

d

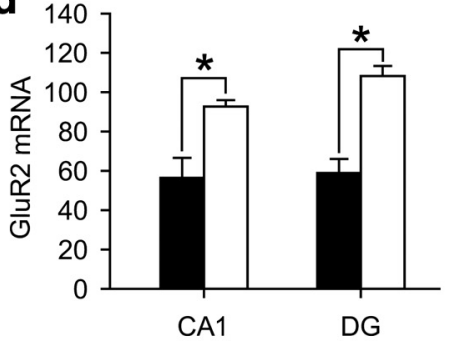

f

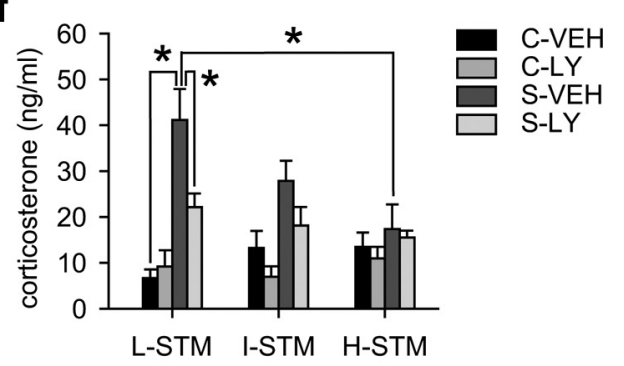

h

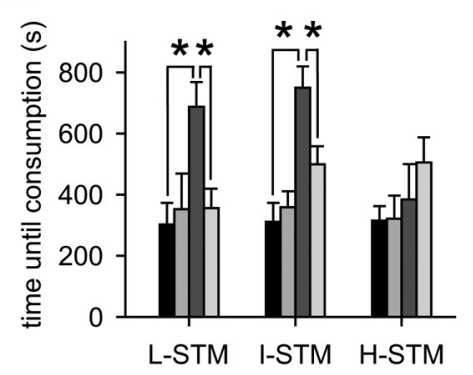

Figure 3. Low STM predicts individual stress vulnerability. $\boldsymbol{a}$, Histogram of spontaneous alteration behavior in the Y-maze test of $\mathrm{CD} 1$ mice at the age of $26-28 \mathrm{~d}(n=140)$. Animals falling in the lowest, highest, or intermediate $20 \%$ of the total group were used for breeding of the L-STM, H-STM, or I-STM line, respectively. $\boldsymbol{b}$, After four generations of selective breeding, the offspring of the three lines differed significantly in terms of STM performance $\left(F 3: F_{(2,122)}=10.225, p<0.0001 ; F 4: F_{(2,158)}=24.693, p<\right.$ 0.0001).c, At postnatal day 28 , animals from the H-STM line displayed significantly lower GluR2 expression in the CA1 and dentate gyrus compared with L-STM animals (CA1: $\left.t_{(10)}=4.183, p<0.01 ; \mathrm{DG}, t_{(10)}=4.419, p<0.001 ; n=6-7\right)$. d, The same difference between H-STM and L-STM animals could be observed in adulthood (CA1: $t_{(10)}=3.699, p<0.01 ; \mathrm{DG}, t_{(10)}=5.834, p<0.001 ; n=$ $6-7) . e$, The F4 offspring was then subjected to 7 weeks of chronic social stress, and half of the animals received treatment with the AMPA receptor potentiator LY451646 during the last 4 weeks of stress exposure. At the end of the stress paradigm, we observed a significant effect of the stress condition $\left(F_{(1,108)}=26.102, p<0.0001\right)$ but no effect of treatment, STM line, or any interaction effects. *Significant difference (control vs stress). $\boldsymbol{f}$, After 5 weeks of recovery under stress- and drug-free conditions, we observed a condition effect $\left(F_{(1,108)}=34.479\right.$, $p<0.0001)$, a treatment effect $\left(F_{(1,108)}=5.912, p<0.05\right)$, and a condition $\times \operatorname{STM}$ line interaction $\left(F_{(2,107)}=5.709, p<0.01\right)$. Posthoc analysis revealed that only animals from the L-STM line displayed significantly elevated corticosterone levels 5 weeks after stress. In addition, only in the L-STM line did we observe a significant effect of the AMPA receptor potentiator treatment in the formerly stressed animals. $\boldsymbol{g}$, We observed a significant negative correlation of STM behavior in the Y-maze at postnatal day 26 (P26) with the corticosterone levels 5 weeks after stress exposure in the vehicle-treated group $(r=-0.404 ; p<0.05 ; n=29)$. $\boldsymbol{h}$, For anxiety-related behavior, we tested the animals 5 weeks after stress exposure in the novelty-induced suppression of feeding test. ANOVA revealed a significant effect of condition $\left(F_{(1,107)}=21.110 ; p<0.0001\right)$ as well as a condition $\times$ treatment interaction $\left(F_{(1,107)}=4.546 ; p<0.05\right)$. A history of chronic social stress exposure increased anxiety only in the L-STM and I-STM groups. In addition, treatment with LY451646 normalized anxietyrelated behavior in L-STM and I-STM individuals but had no additional effect in the H-STM line. DG, Dentate gyrus; $C$, control; S, stress; VEH, vehicle; LY, LY451646. *Significant difference.

procedure and after 5 weeks of recovery $(n=269)$ using a repeated-measures general linear model. In all analyses, stress exposure had a highly significant main effect $(p<0.001)$. Of all tested SNPs, only rs26962886 in GluR1 showed a significant main genotypic effect $\left(F_{(2,263)}=3.34 ; p=\right.$ $0.037)$ as well as a significant interaction of SNP genotype with stress exposure $\left(F_{(2,263)}=3.37 ; p=0.024\right)$, with the interaction term withstanding correction for multiple testing. Post hoc analyses showed that there was a highly significant gene dose-dependent effect on corticosterone levels after 5 weeks of recovery in the stress-exposed $(p=0.0009)$ but not in the control ( $p=0.47)$ group (Fig. $4 c)$. Stress-exposed TT homozygotes showed the highest corticorsterone levels after 5 weeks of recovery. We then tested whether the rs26962886 polymorphism was also associated with a different expression of the GluR1 gene. Homozygous TT allele carriers showed a significantly reduced GluR1 expression in the CA1 and the dentate gyrus subregions of the hippocampus compared with homozygous CC allele carriers (Fig. 4d).

\section{Discussion}

There is by now substantial evidence for the glutamate hypothesis of depression (Hashimoto, 2009), and novel drugs acting on the glutamatergic system show a high potential as antidepressants (Sanacora et al., 2008; Machado-Vieira et al., 2009). However, so far it has not been shown that the glutamatergic system might be crucially involved in individual stress vulnerability, one of the most important factors known to play a major role in the multifactorial etiology of affective disorders. By combining evidence on genetic, pharmacological, and behavioral levels, here we demonstrate for the first time an involvement of hippocampal AMPA receptors in individual stress vulnerability and resilience. Especially the identification of easily accessible parameters that are predictive of individual stress vulnerability open up the unique possibility for preventive treatment.

The glutamatergic system and specifically AMPA receptors have recently become a focus of attention with regard to stress and depression (Alt et al., 2006; Bleakman et al., 2007). Our preclinical data clearly show that individuals with high stress vulnerability also display an altered expression of the AMPA receptor system in the dorsal hippocampus, a region specifically involved in cognitive function (Fanselow and Dong, 2010). Although there are no previous data report- 
ing a potential involvement of the AMPA receptor system in disease vulnerability, the current data are in line with clinical reports, describing that AMPA receptor binding is altered in subjects that committed suicide (Freed et al., 1993). Specifically, a low expression of the subunit GluR1, together with a high expression of the subunit GluR2, seems to be related to psychiatric disorders (Meador-Woodruff et al., 2001). Accordingly, mice lacking the GluR1 subunit, thus with a relatively higher GluR2-to-GluR1 ratio, have recently been postulated as a model of depression (Chourbaji et al., 2008). Furthermore, antidepressants have been shown to modulate AMPA receptor mRNA expression, mRNA editing, and receptor phosphorylation, thereby affecting AMPA receptor expression and function (Barbon et al., 2006; Svenningsson et al., 2007; Sawada et al., 2009). Importantly, elevations of stress hormones per se do not seem to have a direct influence on the mRNA expression of AMPA receptor subunits, (Liu et al., 2006), but rather on AMPA receptor trafficking and mobility (Martin et al., 2009). Together with the data presented here, we can thus hypothesize that an enhancement of AMPA receptor signaling could be one of the therapeutic mechanisms of currently available antidepressants. However, our data should not be generalized, as we focused our analysis of vulnerable animals on HPA axis activity, anxiety-related behavior, and cognition. Although alterations in these parameters are important hallmarks of depression in humans, they only reflect certain aspects of the disease.

AMPA receptor subunit composition critically modulates the receptor properties, and the GluR2 subunit was shown to be a rate-limiting factor for the calcium influx after activation of these receptors, resulting in a desensitization of GluR2-

containing AMPA receptors (for review, see Isaac et al., 2007). Our data suggested a higher total number of available AMPA receptors in stress-vulnerable animals with a subunit composition favoring lower receptor sensitivity. We therefore hypothesized that AMPA signaling would be reduced in stress-vulnerable animals because of a lower AMPA receptor sensitivity, despite the apparent higher AMPA receptor number. As a result of the rapid desensitization of AMPA receptors, which is likely caused by a high surface mobility (Heine et al., 2008), classic agonists are inapplicable to chronically enhance AMPA receptor function. In contrast, positive allosteric modulators function to enhance AMPA receptor signaling in the presence of glutamate without activating the receptors on their own (Arai and Kessler, 2007). Furthermore, the so-called AMPA receptor potentiators or ampakines have recently been shown to have antidepressant properties (Li et al., 2001; Bleakman et al., 2007) and have been discussed as a treatment option for depression (O’Neill and Witkin, 2007). These drugs potentiate AMPA-mediated currents by slowing receptor deactivation and at- tenuating receptor desensitization (Arai and Kessler, 2007). The ampakine LY451646 and its enantiomer LY404187 have been demonstrated to selectively affect the sensitivity of central AMPA receptors and to enhance synaptic excitation in vitro and in vivo (Gates et al., 2001; Miu et al., 2001; Vandergriff et al., 2001; Quirk and Nisenbaum, 2002). Similar to current antidepressants, AMPA potentiation has been shown to increase cell proliferation and survival in the hippocampus (Su et al., 2009). We could now demonstrate that these drugs are also highly effective to reduce individual stress vulnerability and to promote stress resilience and recovery. Whereas this pharmacological effect is no clear evidence for the direct involvement of AMPA receptors in stress vulnerability, our data support previous studies using the same compounds, which showed a decreased depression-like behavior in the tail suspension test and the forced-swim test in a dosedependent manner (Bai et al., 2001). However, the doses used in the study by Bai et al. (2001) were substantially higher than what we have used, thereby supporting an effect of AMPA receptor 
enhancement even at subthreshold doses. This is in line with previous reports, in which the structurally related ampakine LY392098 was shown to increase the efficacy of antidepressants at low doses (Li et al., 2003). Furthermore, it was reported that chronic treatment with AMPA potentiators results in increased hippocampal cell proliferation and antidepressant-like effects in a chronic mild stress paradigm (Bai et al., 2003; Farley et al., 2010). Together, the enhancement of AMPA receptor function seems to be beneficial for individuals with a predisposition for low GluR1 and/or high GluR2 expression and therefore increased stress vulnerability.

There is already ample evidence that AMPA receptors are critically involved in spatial STM (Izquierdo et al., 2002; Schmitt et al., 2005; Sanderson et al., 2009). Interestingly, a pharmacologically induced increase in GluR2 mRNA expression has been shown to correlate with impaired spatial working memory ( $\mathrm{Si}$ moes et al., 2007). We had therefore hypothesized that alterations in AMPA receptor subunit expression should be detectable on a behavioral level. Indeed, we could demonstrate that individuals with the lowest spatial STM performance had a significantly increased risk to be in the group of stress-vulnerable individuals. Intriguingly, specifically those individuals with a low STM showed an increased hippocampal GluR2 expression and responded well to the treatment with an AMPA receptor potentiator. A recent report on abnormalities in cortical activity related to working memory in individuals with high familiar risk for depression indeed suggests working memory as a potential biomarker for individual vulnerability (Mannie et al., 2010). Thus, our data suggest that AMPA receptor function as reflected by the performance in spatial working memory could be a valid biomarker for individual stress vulnerability, which can be pharmacologically counteracted or even prevented by enhancing AMPA receptor function during chronic stress exposure.

Depression is a highly complex disease that has been shown to include a substantial heritable, thus genetic, portion. Kendler et al. (1995) estimated the heritability of depression to be in the range of $30-50 \%$, depending on sex and symptom severity. Here, we could demonstrate that a polymorphism in the mouse GluR1 gene is significantly correlated with individual stress vulnerability. Corresponding to our previous findings, the vulnerable risk allele carriers of this polymorphism also showed a lower hippocampal GluR1 expression, enabling the prediction of relative GluR1 expression and, consequently, stress vulnerability based on a genetic trait. Although the current approach is still limited by the availability of known polymorphisms in the CD1 outbred strain, the example of the GluR1 polymorphism identified here illustrates that genetic variations can indeed have a direct effect on basal gene expression, possibly underlying individual stress vulnerability as well as pharmacological responsivity. Concordantly, diseases such as MDD may emerge as a consequence of individual genetic variability, in combination with severe environmental challenges as chronic stress exposure.

Together, the integration of data from various different levels, including genetic analysis, unbiased microarray profiling, pharmacology, and behavior, point to AMPA receptors as one potential molecular cause for individual stress vulnerability. Our findings suggest that these heritable individual differences can be predicted by easily accessible biomarkers and may serve as novel therapeutic targets for stress-related human disorders.

\section{References}

Alt A, Nisenbaum ES, Bleakman D, Witkin JM (2006) A role for AMPA receptors in mood disorders. Biochem Pharmacol 71:1273-1288.
Arai AC, Kessler M (2007) Pharmacology of ampakine modulators: from AMPA receptors to synapses and behavior. Curr Drug Targets 8:583-602.

Bai F, Li X, Clay M, Lindstrom T, Skolnick P (2001) Intra- and interstrain differences in models of "behavioral despair." Pharmacol Biochem Behav 70:187-192.

Bai F, Bergeron M, Nelson DL (2003) Chronic AMPA receptor potentiator (LY451646) treatment increases cell proliferation in adult rat hippocampus. Neuropharmacology 44:1013-1021.

Barbon A, Popoli M, La Via L, Moraschi S, Vallini I, Tardito D, Tiraboschi E, Musazzi L, Giambelli R, Gennarelli M (2006) Regulation of editing and expression of glutamate [alpha]-amino-propionic-acid (AMPA)/kainate receptors by antidepressant drugs. Biol Psychiatry 59:713-720.

Bleakman D, Alt A, Witkin JM (2007) AMPA receptors in the therapeutic management of depression. CNS Neurol Disord Drug Targets 6:117-126.

Charney DS (1998) Monoamine dysfunction and the pathophysiology and treatment of depression. J Clin Psychiatry 59 [Suppl 14]:11-14.

Chourbaji S, Vogt MA, Fumagalli F, Sohr R, Frasca A, Brandwein C, Hörtnagl H, Riva MA, Sprengel R, Gass P (2008) AMPA receptor subunit 1 (GluR-A) knockout mice model the glutamate hypothesis of depression. FASEB J 22:3129-3134.

Chudin E, Kruglyak S, Baker SC, Oeser S, Barker D, McDaniel TK (2006) A model of technical variation of microarray signals. J Comput Biol 13:996-1003.

Datson NA, Meijer L, Steenbergen PJ, Morsink MC, van der LS, Meijer OC, De Kloet ER (2004) Expression profiling in laser-microdissected hippocampal subregions in rat brain reveals large subregion-specific differences in expression. Eur J Neurosci 20:2541-2554.

Du J, Gray NA, Falke CA, Chen W, Yuan P, Szabo ST, Einat H, Manji HK (2004) Modulation of synaptic plasticity by antimanic agents: the role of AMPA glutamate receptor subunit 1 synaptic expression. J Neurosci 24:6578-6589.

Fanselow MS, Dong HW (2010) Are the dorsal and ventral hippocampus functionally distinct structures? Neuron 65:7-19.

Farley S, Apazoglou K, Witkin JM, Giros B, Tzavara ET (2010) Antidepressantlike effects of an AMPA receptor potentiator under a chronic mild stress paradigm. Int J Neuropsychopharmacol 13:1207-1218.

Fluttert M, Dalm S, Oitzl MS (2000) A refined method for sequential blood sampling by tail incision in rats. Lab Anim 34:372-378.

Freed WJ, Dillon-Carter O, Kleinman JE (1993) Properties of [3H]AMPA binding in postmortem human brain from psychotic subjects and controls: increases in caudate nucleus associated with suicide. Exp Neurol 121:48-56.

Gates M, Ogden A, Bleakman D (2001) Pharmacological effects of AMPA receptor potentiators LY392098 and LY404187 on rat neuronal AMPA receptors in vitro. Neuropharmacology 40:984-991.

Hashimoto K (2009) Emerging role of glutamate in the pathophysiology of major depressive disorder. Brain Res Rev 61:105-123.

Heine M, Groc L, Frischknecht R, Beique JC, Lounis B, Rumbaugh G, Huganir RL, Cognet L, Choquet D (2008) Surface mobility of postsynaptic AMPARs tunes synaptic transmission. Science 320:201-205.

Huynh NN, McIntyre RS (2008) What are the implications of the STAR ${ }^{\star} \mathrm{D}$ trial for primary care? A review and synthesis. Prim Care Companion J Clin Psychiatry 10:91-96.

Isaac JTR, Ashby M, McBain CJ (2007) The role of the GluR2 subunit in AMPA receptor function and synaptic plasticity. Neuron 54:859-871.

Izquierdo LA, Barros DM, Vianna MR, Coitinho A, deDavid e Silva, Choi H, Moletta B, Medina JH, Izquierdo I (2002) Molecular pharmacological dissection of short- and long-term memory. Cell Mol Neurobiol 22:269-287.

Kendler KS, Kessler RC, Walters EE, MacLean C, Neale MC, Heath AC, Eaves LJ (1995) Stressful life events, genetic liability, and onset of an episode of major depression in women. Am J Psychiatry 152:833-842.

Kessler RC, Berglund P, Demler O, Jin R, Koretz D, Merikangas KR, Rush AJ, Walters EE, Wang PS (2003) The epidemiology of major depressive disorder: results from the National Comorbidity Survey Replication (NCSR). JAMA 289:3095-3105.

Kim JS, Schmid-Burgk W, Claus D, Kornhuber HH (1982) Increased serum glutamate in depressed patients. Arch Psychiatr Nervenkr 232:299-304.

Li X, Tizzano JP, Griffey K, Clay M, Lindstrom T, Skolnick P (2001) Antidepressant-like actions of an AMPA receptor potentiator (LY392098). Neuropharmacology 40:1028-1033.

Li X, Witkin JM, Need AB, Skolnick P (2003) Enhancement of antidepres- 
sant potency by a potentiator of AMPA receptors. Cell Mol Neurobiol 23:419-430.

Liu HH, Payne HR, Wang B, Brady ST (2006) Gender differences in response of hippocampus to chronic glucocorticoid stress: role of glutamate receptors. J Neurosci Res 83:775-786.

Machado-Vieira R, Manji HK, Zarate CA (2009) The role of the tripartite glutamatergic synapse in the pathophysiology and therapeutics of mood disorders. Neuroscientist 15:525-539.

Mackowiak M, O’Neill MJ, Hicks CA, Bleakman D, Skolnick P (2002) An AMPA receptor potentiator modulates hippocampal expression of BDNF: an in vivo study. Neuropharmacology 43:1-10.

Maeng CA, Zarate CA (2007) The role of glutamate in mood disorders: results from the ketamine in major depression study and the presumed cellular mechanism underlying its antidepressant effects. Curr Psychiatry Rep 9:467-474.

Maeng S, Zarate CA, Du J, Schloesser RJ, McCammon J, Chen G, Manji HK (2008) Cellular mechanisms underlying the antidepressant effects of ketamine: role of alpha-amino-3-hydroxy-5-methylisoxazole-4-propionic acid receptors. Biol Psychiatry 63:349-352.

Mannie ZN, Harmer CJ, Cowen PJ, Norbury R (2010) A functional magnetic resonance imaging study of verbal working memory in young people at increased familial risk of depression. Biol Psychiatry 67:471-477.

Martin S, Henley JM, Holman D, Zhou M, Wiegert O, van Spronsen M, Joels M, Hoogenraad CC, Krugers HJ (2009) Corticosterone alters AMPAR mobility and facilitates bidirectional synaptic plasticity. PLoS One 4:e4714.

Meador-Woodruff JH, Hogg AJ, Smith RE (2001) Striatal ionotropic glutamate receptor expression in schizophrenia, bipolar disorder, and major depressive disorder. Brain Res Bull 55:631-640.

Merali Z, Levac C, Anisman H (2003) Validation of a simple, ethologically relevant paradigm for assessing anxiety in mice. Biol Psychiatry 54:552-565.

Mitani H, Shirayama Y, Yamada T, Maeda K, Ashby CR, Kawahara R (2006) Correlation between plasma levels of glutamate, alanine and serine with severity of depression. Prog Neuropsychopharmacol Biol Psychiatry 30:1155-1158.

Miu P, Jarvie KR, Radhakrishnan V, Gates MR, Ogden A, Ornstein PL, Zarrinmayeh H, Ho K, Peters D, Grabell J, Gupta A, Zimmerman DM, Bleakman D (2001) Novel AMPA receptor potentiators LY392098 and LY404187: effects on recombinant human AMPA receptors in vitro. Neuropharmacology 40:976-983.

O’Neill MJ, Witkin JM (2007) AMPA receptor potentiators: application for depression and Parkinson's disease. Curr Drug Targets 8:603-620.

Ozbay F, Fitterling H, Charney D, Southwick S (2008) Social support and resilience to stress across the life span: a neurobiologic framework. Curr Psychiatry Rep 10:304-310.
Quirk JC, Nisenbaum ES (2002) LY404187: a novel positive allosteric modulator of AMPA receptors. CNS Drug Rev 8:255-282.

Rao VR, Finkbeiner S (2007) NMDA and AMPA receptors: old channels, new tricks. Trends Neurosci 30:284-291.

Sanacora G, Zarate CA, Krystal JH, Manji HK (2008) Targeting the glutamatergic system to develop novel, improved therapeutics for mood disorders. Nat Rev Drug Discov 7:426-437.

Sanderson DJ, Good MA, Skelton K, Sprengel R, Seeburg PH, Rawlins JN, Bannerman DM (2009) Enhanced long-term and impaired short-term spatial memory in GluA1 AMPA receptor subunit knockout mice: evidence for a dual-process memory model. Learn Mem 16:379-386.

Sawada J, Yamashita T, Aizawa H, Aburakawa Y, Hasebe N, Kwak S (2009) Effects of antidepressants on GluR2 Q/R site-RNA editing in modified HeLa cell line. Neurosci Res 64:251-258.

Schmidt MV, Sterlemann V, Ganea K, Liebl C, Alam S, Harbich D, Greetfeld M, Uhr M, Holsboer F, Müller MB (2007) Persistent neuroendocrine and behavioral effects of a novel, etiologically relevant mouse paradigm for chronic social stress during adolescence. Psychoneuroendocrinology 32:417-429.

Schmidt MV, Scharf SH, Sterlemann V, Ganea K, Liebl C, Holsboer F, Müller MB (2009) High susceptibility to chronic social stress is associated with a depression-like phenotype. Psychoneuroendocrinology 35:635-643.

Schmitt WB, Sprengel R, Mack V, Draft RW, Seeburg PH, Deacon RMJ, Rawlins JNP, Bannerman DM (2005) Restoration of spatial working memory by genetic rescue of GluR-A-deficient mice. Nat Neurosci 8:270-272.

Simoes PF, Silva AP, Pereira FC, Marques E, Grade S, Milhazes N, Borges F, Ribeiro CF, Macedo TR (2007) Methamphetamine induces alterations on hippocampal NMDA and AMPA receptor subunit levels and impairs spatial working memory. Neuroscience 150:433-441.

Su XW, Li XY, Banasr M, Koo JW, Shahid M, Henry B, Duman RS (2009) Chronic treatment with AMPA receptor potentiator Org 26576 increases neuronal cell proliferation and survival in adult rodent hippocampus. Psychopharmacology 206:215-222.

Svenningsson P, Bateup H, Qi H, Takamiya K, Huganir RL, Spedding M, Roth BL, McEwen BS, Greengard P (2007) Involvement of AMPA receptor phosphorylation in antidepressant actions with special reference to tianeptine. Eur J Neurosci 26:3509-3517.

Vandergriff J, Huff K, Bond A, Lodge D (2001) Potentiation of responses to AMPA on central neurones by LY392098 and LY404187 in vivo. Neuropharmacology 40:1003-1009.

Wang J (2005) Work stress as a risk factor for major depressive episode(s). Psychol Med 35:865-871.

Zarate CA, Singh JB, Carlson PJ, Brutsche NE, Ameli R, Luckenbaugh DA, Charney DS, Manji HK (2006) A randomized trial of an N-methyl-Daspartate antagonist in treatment-resistant major depression. Arch Gen Psychiatry 63:856-864. 